\title{
Noninvasive probing of persistent conductivity in high quality ZnCdSe/ZnSe quantum wells using surface acoustic waves
}

Cite as: J. Appl. Phys. 107, 093717 (2010); https://doi.org/10.1063/1.3373415

Submitted: 08 December 2009. Accepted: 28 February 2010 . Published Online: 07 May 2010

D. A. Fuhrmann, H. J. Krenner, A. Wixforth, A. Curran, K. A. Prior, R. J. Warburton, and J. Ebbecke

\section{ARTICLES YOU MAY BE INTERESTED IN}

Independent dynamic acousto-mechanical and electrostatic control of individual quantum dots in a LiNbOz-GaAs hybrid

Applied Physics Letters 106, 013107 (2015); https://doi.org/10.1063/1.4905477

Direct observation of dynamic surface acoustic wave controlled carrier injection into single quantum posts using phase-resolved optical spectroscopy

Applied Physics Letters 98, 023109 (2011); https://doi.org/10.1063/1.3541881

Combined electrical transport and capacitance spectroscopy of a $\mathrm{MoS}_{2}-\mathrm{LiNbO}_{3}$ field effect transistor

Applied Physics Letters 110, 023505 (2017); https://doi.org/10.1063/1.4973862

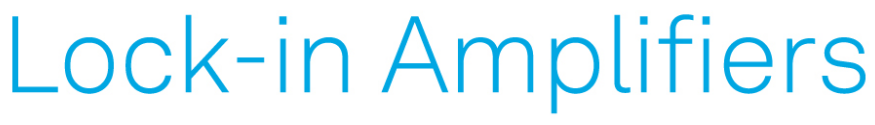
... and more, from DC to $600 \mathrm{MHz}$

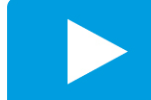

Watch 


\title{
Noninvasive probing of persistent conductivity in high quality ZnCdSe/ ZnSe quantum wells using surface acoustic waves
}

\author{
D. A. Fuhrmann, ${ }^{1}$ H. J. Krenner, ${ }^{1, a)}$ A. Wixforth, ${ }^{1,2}$ A. Curran, ${ }^{3}$ K. A. Prior, ${ }^{3}$ \\ R. J. Warburton, ${ }^{3,4}$ and J. Ebbecke ${ }^{2,5}$ \\ ${ }^{1}$ Institut für Physik der Universität Augsburg, Experimentalphysik I, Universitätsstr. 1, \\ 86159 Augsburg, Germany \\ ${ }^{2}$ Center for NanoScience (CeNS), Geschwister-Scholl-Platz 1, 80539 Munich, Germany \\ ${ }^{3}$ School of Engineering and Physical Sciences, Heriot-Watt University, Edinburgh, EH14 4AS, \\ United Kingdom \\ ${ }^{4}$ Department of Physics, University of Basel, Klingelbergstr. 82, 4056 Basel, Switzerland \\ ${ }^{5}$ University of Southern Denmark, Mads Clausen Institute, NanoSYD, Alsion 2, DK-6400 Sфnderborg, \\ Denmark
}

(Received 8 December 2009; accepted 28 February 2010; published online 7 May 2010)

\begin{abstract}
Attenuation of a surface acoustic wave is used as a highly sensitive and noninvasive probe of persistent photoconductivity effects in $\mathrm{ZnCdSe} / \mathrm{ZnSe}$ quantum wells. These effects are observed over long time-scales exceeding several minutes at low temperatures. By varying the optical excitation energy and power and temperature we show that these effects arise from carriers photogenerated by interband excitation which are trapped in random potential fluctuations in the quantum wells related to compositional fluctuations. Effects related to defect levels in the band gap can be excluded and a transition of the conduction mechanism with temperature from a hopping to a percolation regime is observed. The transition temperature observed for our quantum well material is strongly reduced compared to bulk crystals. This indicates a superior structural quality giving rise to only weak potential fluctuation of $\lesssim 3 \mathrm{meV}$. (C) 2010 American Institute of Physics.
\end{abstract}

[doi:10.1063/1.3373415]

\section{INTRODUCTION}

Surface acoustic waves (SAW) are a highly versatile tool to probe and manipulate optical and electronic properties of materials. Examples in the field of low-dimensional semiconductor heterostructures include charge conveyance and spectroscopy of high-mobility electron systems ${ }^{1,2}$ and optically active systems as quantum wells $(\mathrm{QWs})^{3,4}$ and quantum dots. ${ }^{5-7}$ However, most of these investigations focused on the most advanced III-V semiconductor compounds due to their piezoelectricity which allows for the use of interdigital transducers (IDTs) to excite and detect SAW. Despite the fact that most II-VI semiconductors are also weakly piezoelectric still SAW-based studies are very limited. ${ }^{8}$

One important mechanism which has large impact on the electrical transport properties in semiconductors is the persistent photoconductivity effect (PPC). This persistence of free charge carriers in semiconductors long after the removal of the exciting light source can by far exceed typical carrier lifetimes and leads to a pronounced change of conductivity in the material. PPC has been studied in the AlGaAs and other III-V material systems in great detail for many years. In the case of $\mathrm{AlGaAs}$ a thermal barrier prevents the recombination of the DX-centers at low temperatures which gives rise to the PPC effect. Similar PPC effects have also been observed in II-VI semiconductors such as $\mathrm{ZnCdSe}$, $\mathrm{ZnMgSSe}, \mathrm{ZnMgSe}$, and CdTe. ${ }^{10,11}$ In inhomogeneous semiconductors ${ }^{12}$ and heterostructures ${ }^{13}$ its origin can be of a different nature. In particular for bulk $\mathrm{ZnCdSe}$ spatial sepa-

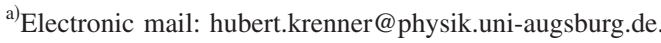

ration of charge carriers in random local potential fluctuations (RLPF) can occur. These RLPF result from compositional fluctuations during material growth and have been found to be the dominant mechanism giving rise to PPC. ${ }^{14-20}$ One striking consequence is the transition of the conduction mechanism from a localized to a percolation regime at a critical temperature. Surprisingly, similar transition temperatures were found for both bulk and QWs grown by molecular beam epitaxy (MBE) (Ref. 21) indicating that in these materials RLPFs are introduced inherently and cannot be reduced by introducing heterointerfaces.

In this paper we present a study of PPC in a $\mathrm{ZnCdSe} /$ ZnSe multiple QW (MQW) structure without directly measuring the conductivity by electrical means. Instead we apply a method based on surface acoustic SAWs, which for low SAW power represents a noninvasive and highly sensitive probe of the conductivity. The underlying mechanism is the attenuation of the SAW by mobile carriers which can be measured with high precision. Moreover, we find that for our QW material the transition temperature between the localized and percolation transport regime is significantly reduced to $T_{C} \lesssim 40 \mathrm{~K}$ compared to values of $T_{C}^{\text {bulk }} \sim 120 \mathrm{~K}$ reported for bulk material. ${ }^{16}$ This finding clearly shows the superior quality of our material compared to previous reports and indicates that inhomogeneities resulting in RLPFs are substantially reduced.

\section{EXPERIMENTAL SETUP}

Our sample consists of a $\mathrm{ZnCdSe} / \mathrm{ZnSe}$ heterostructure grown by MBE. Five optically active, $4 \mathrm{~nm}$ wide 


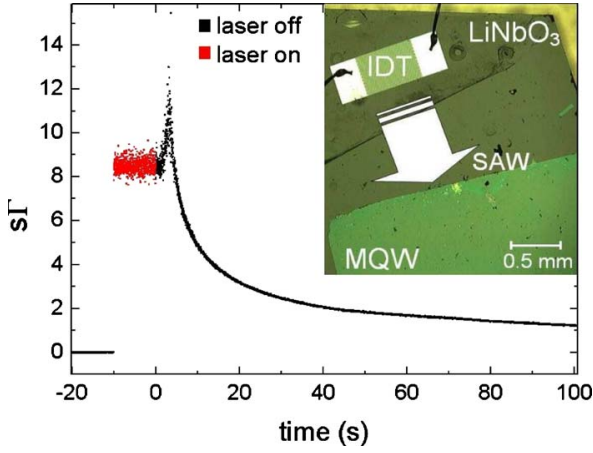

FIG. 1. (Color online) SAW attenuation of the third SAW harmonic $\left(f_{3}=345 \mathrm{MHz}\right)$ at $T=35 \mathrm{~K}$, before, during, and after optical excitation. Inset: $\mathrm{ZnCdSe} / \mathrm{ZnSe}$ heterostructure on a $\mathrm{LiNbO}_{3}$ substrate with IDT.

$\mathrm{Zn}_{0.9} \mathrm{Cd}_{0.1} \mathrm{Se} \mathrm{QWs}$, separated by $8 \mathrm{~nm} \mathrm{ZnSe}$ barriers are embedded between two $63 \mathrm{~nm} \mathrm{ZnSe}$ cladding layers. After growth, the II-VI semiconductor heterostructure was removed from the substrate and transferred onto the strongly piezoelectric $\mathrm{LiNbO}_{3}$ chip using an epitaxial lift-off method. ${ }^{8,22}$ The transferred film is positioned between two IDTs on the host $\mathrm{LiNbO}_{3}$ substrate. Due to the increased coupling coefficient, $K_{\text {eff }}$, of $\mathrm{LiNbO}_{3}$ compared to $\mathrm{Zn}(\mathrm{Cd}) \mathrm{Se}$ this hybrid sample layout efficiently gives access to higher piezoelectric fields in the semiconductor material which, for e.g., was used to study SAW mediated exciton dissociation. ${ }^{8}$ A microscope image of one part of the sample is shown in the inset to Fig. 1. In the upper part the IDT with an aperture of $0.58 \mathrm{~mm}$ allows for the excitation and/or detection of SAW at $f_{0}=115 \mathrm{MHz}$ and $f_{3}=3 \times f_{0}$. The SAW launched by the IDT propagates through the semiconductor film (length $2.6 \mathrm{~mm}$ ) to a second IDT on the opposite side. The total length of the SAW delay line is $5.4 \mathrm{~mm}$ and the transmitted power was measured using a conventional network analyzer. The sample itself was mounted on the coldfinger of an optical helium-flow cryostat equipped with high-frequency connections to the IDTs. For photoluminescence (PL) experiments carriers were photogenerated by a laser diode (Laser-1, $\hbar \omega_{e x}=3.18 \mathrm{eV}, \quad P_{\max }=30 \mathrm{~mW}$ ) focused to $\sim 500 \mu \mathrm{m}$ diameter spot using a $5 \times$ microscope objective. The corresponding maximum excitation density results to $I_{0}=4 \mathrm{~W} \mathrm{~cm}^{-2}$. The emission from the sample was collected by the same objective, sent to a $0.5 \mathrm{~m}$ grating monochromator where it is spectrally analyzed and detected by a $l \mathrm{~N}_{2}$ cooled charge-coupled device. For excitation energy dependent experiments Laser-1 was replaced by white light spectrally filtered by a monochromator. For optical manipulation of the PPC a second laser diode (Laser- $2, \hbar \omega_{e x}=1.84 \mathrm{eV}$, $P_{\max }=35 \mathrm{~mW}$ ) was used.

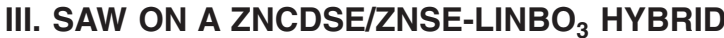

SAW are attenuated in the presence of free charge carriers close to the sample surface. In a piezoelectric material the periodic mechanical deformation is accompanied by electric fields which induce currents dissipating energy. While for high SAW intensities $\left(P_{\mathrm{SAW}}>20 \mathrm{dBm}\right)$ a modulation of the conduction and valence band (VB) edges leads to a dissociation of optically generated excitons, thus quenching the photoluminescence, ${ }^{8}$ in the regime of low powers $P_{\mathrm{SAW}}=-20 \mathrm{dBm}$, used in all presented measurements, the photoluminescence signal of the QW is not yet altered or quenched. The SAW propagation hence acts only as a weak perturbation of the system and a measurement of the SAW transmission in this power range provides a noninvasive probe of the conductivity. The transmitted intensity between the two IDTs is given by $I=I_{0, \mathrm{SAw}} e^{-s \Gamma}$, with $s$ being the length of the interaction region and $\Gamma$ the absorption coefficient. For a two-dimensional system ${ }^{1}$ and small SAW amplitudes, the SAW has only a negligible effect on the charge density $\rho(\mathbf{r}, t)$, therefore, for a given sheet conductivity, $\sigma$, the absorption coefficient, $\Gamma$, can be written as

$$
\Gamma=\frac{K_{\mathrm{eff}}^{2} \cdot k}{2} \times \frac{\frac{\sigma}{\sigma_{m}}}{1+\left(\frac{\sigma}{\sigma_{m}}\right)^{2}} .
$$

In this equation $K_{\text {eff }}$ denotes the effective coupling constant, a measure of the piezoelectric strength of the material, $k$ the SAW wave vector, and $\sigma_{m}$ the so-called characteristic conductivity. From a closer examination of Eq. (1) one can see that the absorption coefficient as a function of conductivity increases linearly for $\sigma<\sigma_{m}$, reaches a maximum at $\sigma=\sigma_{m}$ and subsequently decreases proportional to $\sigma^{-1}$ with further increasing conductivity. The characteristic conductivity $\sigma_{m}$ is given by $\sigma_{m}=\epsilon_{0}\left(1+\epsilon_{\text {hyb }}\right) v$ and is thus dependent on the speed of sound $v$ in the material along the respective crystal direction and the relative dielectric constant of the hybrid system. $\epsilon_{\text {hyb }}$ can be determined for a given layer sequence ${ }^{23}$ and depends on the SAW wave vector and, therefore, the different harmonics used in our experiments exhibit different values for the characteristic conductivities

$$
\begin{aligned}
& \sigma_{m}(115 \mathrm{MHz}) \approx 1.04 \times 10^{-6} \Omega^{-1}, \\
& \sigma_{m}(345 \mathrm{MHz}) \approx 8.75 \times 10^{-7} \Omega^{-1} .
\end{aligned}
$$

At these critical conductivities pronounced minima in the transmitted SAW power for both frequencies are expected. The small values for $\sigma_{m}$ indicate the high sensitivity of the SAW to measure small conductivities. In the following the measured parameter will be $s \Gamma$, the length of the interaction region $s$ multiplied by the absorption coefficient, referred to as the SAW attenuation. While the upper bound of $s$ is determined by the size of the epitaxial lift-off film $(2.6 \mathrm{~mm})$ the effective value of $s$ over which changes in the conductivity are detected is mainly limited to the excitation spot size of $\sim 0.5 \mathrm{~mm}$. Since we keep the spot size constant in our experiments, $s \Gamma$ is a direct measure for the conductivity which is linked by Eq. (1).

Figure 1 shows a typical measurement of attenuation at the frequency of the third harmonic $\left(f_{3}=345 \mathrm{MHz}\right)$ at low temperatures $(T=35 \mathrm{~K})$ as a function of time. At the onset of illumination $(t=-10 \mathrm{~s})$ the attenuation instantly rises. Due to the creation of free charge carriers, the transmission of the $\mathrm{SAW}$ is attenuated by $\approx 35 \mathrm{~dB}$. At $t=0 \mathrm{~s}$ the laser is turned off, after which the attenuation rises to a maximum, before it very slowly decays on a seconds timescale. This behavior is 


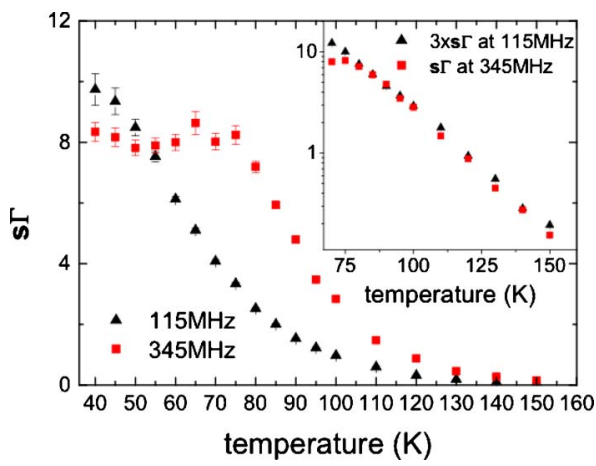

FIG. 2. (Color online) Temperature dependence of the attenuation of the first and third SAW harmonic on illumination. Inset: Measured values of the first harmonic are multiplied by three, to show a $s \Gamma \propto k$ dependence for $\sigma<\sigma_{m}$, i.e., at low temperatures.

consistent with Eq. (1). In particular, at the maximum of the attenuation the conductivity is equal to the characteristic conductivity $\sigma_{m}$.

The increase in the attenuation during illumination as a function of temperature is shown in Fig. 2. For $T>150 \mathrm{~K}$ the transmission of the SAW is not damped and no attenuation is measured. As this is the same temperature above which no photoluminescence could be detected for our sample, ${ }^{8}$ it hints toward charge carriers from band-to-band transitions as the origin of the PPC. The attenuation of the third harmonic shows a nonlinear dependence on the conductivity for $T<70 \mathrm{~K}$ as described by Eq. (1). At this temperature the conductivity is very close to the characteristic conductivity $\sigma_{m} \approx 8.75 \times 10^{-7} \Omega^{-1}$ of the third harmonic. Since the value of the characteristic conductivity of the first harmonic is higher, the attenuation remains directly proportional to the conductivity over the entire range of temperatures $T$ $>10 \mathrm{~K}$. Unless stated otherwise we restrict our analysis to data obtained at $f_{1}$ due to the linear dependency of the conductivity on the SAW attenuation. For $T>75 \mathrm{~K}$ the measured values of the third SAW harmonic match those of the first multiplied by three (see inset to Fig. 2). This indicates that the slight difference of $\sigma_{m}(345 \mathrm{MHz})$ and $\sigma_{m}(115$ $\mathrm{MHz}$ ) expresses itself in the attenuation for conductivities in the range of $\sigma_{m}$ and shows very nicely that the attenuation is a relative measure of sheet conductivity.

\section{A. PCC in ZnCdSe QWs}

As shown in Fig. 1, the SAW propagation is considerably affected during and long after illumination. This is a rather striking discovery, because in an ideal system in which only charge neutral excitons are generated, the dominant loss mechanism would be radiative recombination. This process takes place on much faster timescales which are not affected by the small amplitudes of the SAW (Ref. 3) used in these experiments. In order to exclude optically active impurities giving rise to levels in the band gap or surface states, the SAW attenuation was investigated for different photon energies. In this experiment Laser-1 was replaced by white light which was spectrally filtered by our monochromator. Figure 3 shows the SAW attenuation of the first harmonic during illumination at $T=60 \mathrm{~K}$ as a function of the photon energy. At this temperature the attenuation is directly proportional to

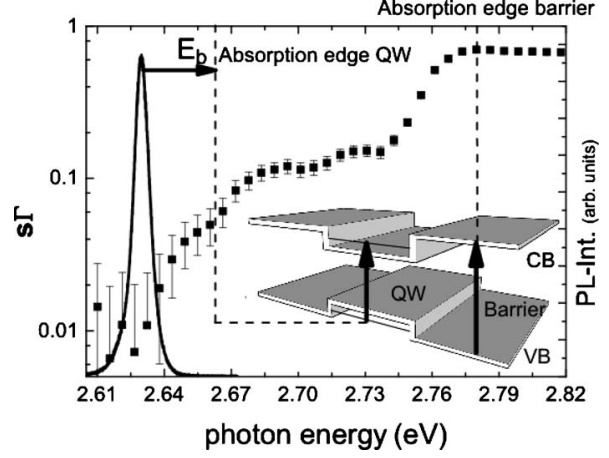

FIG. 3. SAW attenuation and free exciton PL-intensity at $T=60 \mathrm{~K}$. The energy of the free exciton recombination and the exciton binding energy $E_{b} \approx 32.5 \mathrm{meV}$ (Ref. 24) determine the onset of free charge carrier generation in the QW. The energy for the absorption edge of $\mathrm{ZnSe}$ is approximately $2.78 \mathrm{eV}$ at $60 \mathrm{~K}$ (Ref. 25). The inset shows a schematic of the conduction band $(\mathrm{CB})$ and $\mathrm{VB}$ of $\mathrm{ZnCdSe}-\mathrm{QW}$ and $\mathrm{ZnSe}$-barrier in order to illustrate the origin of the attenuating charge carriers (not to scale).

the conductivity, as indicated by the temperature dependence of the first SAW harmonic shown in Fig. 2 and, moreover, the low optical powers used in this experiment ensure that the conductivity is much less $\sigma_{m}$. Photon energies less or equal than the free exciton recombination energy (a PL spectrum is shown in the figure for comparison), $\hbar \omega \approx 2.63 \mathrm{eV}$, have no effect on the SAW transmission. This excludes activation of defects or impurities with levels within the band gap as the mechanism responsible for the observed effect as it was observed in a previous study on II-VI QWs grown by MBE. ${ }^{21}$ For energies larger than the QW emission two regions can be distinguished: (i) for energies ranging between 2.63 and $\sim 2.78 \mathrm{eV}$ charge carriers are photogenerated solely in the $\mathrm{ZnCdSe}-\mathrm{QW}$. (ii) For energies exceeding the band gap of the $\mathrm{ZnSe}$ barrier material, $E_{g}(\mathrm{ZnSe})$ $\approx 2.78 \mathrm{eV},{ }^{25}$ carriers are also generated outside the QW region. The increase in the signal at this absorption edge indicates that some of the carriers excited in the barrier material relax into the QWs levels.

To obtain further insight in the underlying mechanism we performed temperature dependent SAW attenuation and PL experiments under illumination by Laser-1 which are summarized in Fig. 4. In the SAW attenuation experiments the third harmonic was used [Fig. 4(a)] and the laser was switched off at $t=0 \mathrm{~s}$. Clearly, the time transients undergo a pronounced change as the temperature is raised. In particular the peak in the SAW attenuation at which the conductivity becomes comparable to $\sigma_{m}$ shifts in time and its temporal width changes. At a temperature of $T \sim 30-40 \mathrm{~K}$ both the delay and width of this conductivity peak are maximum. For lower and higher temperatures this peak vanishes and, in addition, a slow and fast decay of the attenuation signal are observed, respectively. Since the observed maximum is linked to a shift of $\sigma$ from values larger to values smaller than $\sigma_{m}$ its width and contrast reflect the recombination mechanism and rate of the charge carriers in the system. Though $\sigma(t=0 \mathrm{~s})$ is not the same for all temperatures the temporal width of the attenuation peak is directly proportional to the recombination rate and these measurements suggest a minimum recombination rate at $T \sim 30 \mathrm{~K}$. Thus a critical temperature $T_{C}$ is exceeded in the range between 

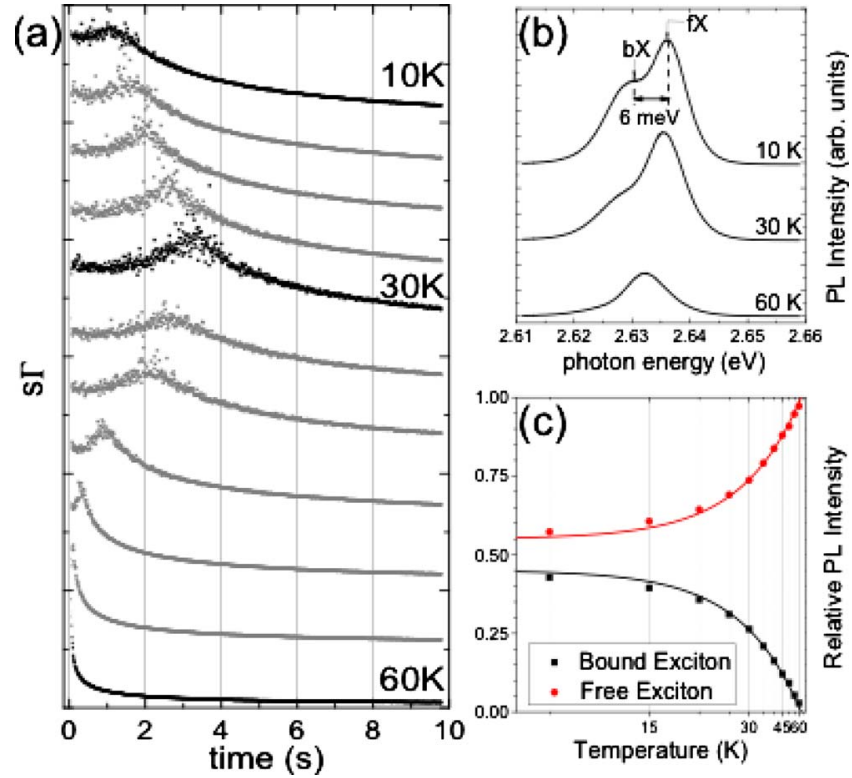

FIG. 4. (Color online) (a) Decay of attenuation of the third SAW harmonic for temperatures $T=10-60 \mathrm{~K}(\Delta T=5 \mathrm{~K})$. (b) PL spectra at $T=10,35$, and $60 \mathrm{~K}$ showing bound exciton (bX) and free exciton (fX) luminescence. (c) Relative PL intensity of free and bound exciton (symbols) and fit of Eqs. (2) and (3) (solid lines).

$25<T<35 \mathrm{~K}$ above which the recombination rate increases rapidly with temperature and exhibits an activated temperature dependence.

This critical temperature corresponds to a thermal energy of $k_{B} T_{C}=2.6 \mathrm{meV}$ which suggests an activation energy in the same range. Since the excitation energy dependence of the PPC indicates that the underlying mechanism is governed by carriers photogenerated across the bandgap we can use PL spectroscopy to obtain further information over the same temperature range. In Fig. 4(b) we compare the QW emission at $T=10,30$, and $60 \mathrm{~K}$ which are below, close to and above the temperature at which the transition of the SAW attenuation transients is observed. For clarity the corresponding SAW transients at these temperatures are highlighted in Fig. 4(a). A close examination of the PL signal at $T=10 \mathrm{~K}$ shows two contributions of similar intensity which are split by $\sim 6 \mathrm{meV}$. The features at lower and higher energy arise from emission of bound (bX) and free excitons (fX), respectively. While for $T=30 \mathrm{~K} \mathrm{bX}$ is suppressed compared to $\mathrm{fX}$ but still well resolved at $T=60 \mathrm{~K}$ only $\mathrm{fX}$ emission is detected. For a detailed analysis of this effect in the temperature range between $T=10$ and $60 \mathrm{~K}$ we plot the relative intensities of bX and fX given by $I_{r e l, \mathrm{bX} / \mathrm{fX}}=I_{\mathrm{bX} / \mathrm{fX}} / I_{\mathrm{bX}}+I_{\mathrm{fX}}$ as symbols in Fig. 4(c). This data can be described by a thermally activated conversion process from bound (bX) into free excitons (fX)

$$
\begin{aligned}
& I_{r e l, \mathrm{bX}}(T)=\frac{I_{\mathrm{bX}}}{I_{\mathrm{bX}}+I_{\mathrm{fX}}}=1-\left[\exp \left(\frac{-\Delta E}{k_{B} T}\right)+\alpha\right], \\
& I_{r e l, \mathrm{fX}}(T)=\frac{I_{\mathrm{fX}}}{I_{\mathrm{bX}}+I_{\mathrm{fX}}}=\exp \left(\frac{-\Delta E}{k_{B} T}\right)+\alpha .
\end{aligned}
$$

Here, $\Delta E$ is the exciton localization energy and $\alpha$ is a constant which reflects the number of free excitons for $T$

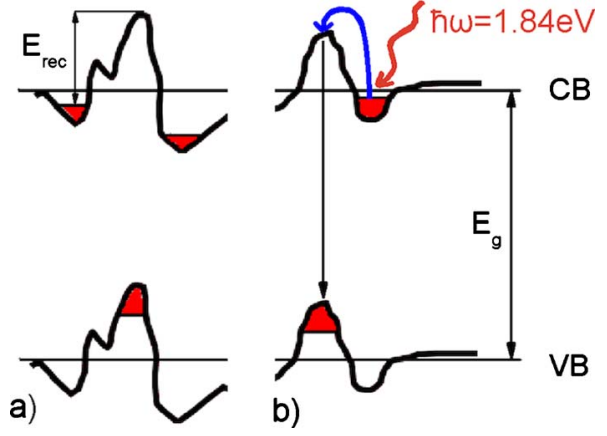

FIG. 5. (Color online) (a) Schematic of spatial modulation of the conduction and VB edge energies due to RLPFs with a recombination energy $E_{\text {rec }}$. Horizontal lines labeled $\mathrm{CB}$ and VB show the nominal conduction and VB edges without RLPF. (b) Excitation of trapped charge carriers using subband gap excitation.

$\rightarrow 0 \mathrm{~K}$ for a fixed generation rate, i.e., optical pump power. The corresponding fits of Eqs. (2) and (3) are plotted as solid lines in Fig. 4(c). From these fits we obtain a localization energy $\Delta E=4.3 \mathrm{meV}$, a value which is comparable to and consistent with the energy splitting between the $\mathrm{bX}$ and $\mathrm{fX}$ peaks. Moreover, the thermal energy $k_{B} T_{C}=2.6 \mathrm{meV}$ at which the transition is observed in the SAW attenuation is also in good agreement. Since both SAW attenuation and PL spectroscopy show a transition in the same temperature range we assume that also for the PPC measured via the SAW attenuation localization of carriers is the dominant mechanism.

In a semiconductor alloy disorder can lead to compositional variations and the resulting variations in the conduction and VB edges give rise to RLPF. These RLPF can act as local traps for charge carriers as shown schematically in Fig. 5. For our $\mathrm{ZnCdSe} \mathrm{MQW}$, local chemical fluctuations, i.e., of the Cd-content can create potential minima for either electrons or holes, which exhibit an average energy barrier $E_{\text {rec }}$ which is a measure for the disorder in the system. Moreover, to transfer carriers between RLPF or release excitons bound to an RLPF the corresponding recombination energy $E_{\text {rec }}$ has to be provided, for example, thermally which gives rise to the observed temperature dependence. For low temperatures, charge carriers are localized in all potential minima, thus the resulting conductivity is very low. The separation leads to a reduced wave function overlap of electrons and holes inhibiting, e.g., radiative recombination. This consequently results in an increase in the carrier lifetime giving rise to PPC. With increasing thermal energy or temperature, thermal redistribution of carriers sets in. In particular at a critical temperature $T_{C}$, a transition of the conduction mechanism occurs, ${ }^{17}$ carriers can now percolate through the network of accessible potential minima. At this temperature this percolation leads to a net transfer of carriers to the deepest RLPF. This increases the average carrier lifetime giving rise to the slow down of the decay of the PPC [see measurement in Fig. 4(a)] for temperatures up to $T=30 \mathrm{~K}$. With further increasing temperature, the recombination rate rises until $E_{\text {rec }}$ becomes comparable to the thermal energy. From this temperature on PPC breaks down and is no longer observed since now all 
carriers trapped in RLPF are thermally activated out of these localization sites. This increases the recombination rate and the PPC decays faster.

The good agreement between $E_{\text {rec }}$, i.e., the depth of the RLPF determined using our SAW-based method and the exciton localization energy determined from the PL data further confirms that both measured energies reflect the bandedge modulation. These small measured localization energies reflect directly the excellent structural and morphological quality of our MBE grown QWs.

As discussed in the previous paragraph, the critical temperature $T_{C}$ at which the transition of the transport mechanism occurs depends on the depth of the energy minima. These themselves are directly connected to disorder in the material composition and thus the sample quality. Therefore, a small value of $T_{C}$ indicates that there is a weak and shallow potential modulation due to RLPF. In previous PPC studies on II-VI semiconductor compounds RLPF have been identified in bulk material to be the dominant mechanism at play. The reported values of $T_{C}>120 \mathrm{~K}$ were found for bulk crystals $^{16}$ and MBE-grown QWs. ${ }^{21}$ These studies indicate that the introduction of a heterointerface as in a QWs does not necessarily result in a reduction in RLPF. Comparing the findings of Ref. 21 to our work we do not observe PPC effects for sub-band gap excitation which indicates that RLPF are the dominant mechanism. Moreover, from the presented data we can deduce a value of $T_{C}<40 \mathrm{~K}$ for our sample. This strong reduction in the critical temperature and the corresponding shallow band edge modulation of $E_{\mathrm{rec}}$ $\$ 3 \mathrm{meV}$ underlines the high quality of our films. This value is comparable to the activation energy between bound and free excitons of $\sim 4.3 \mathrm{meV}$. The small deviation could arise from different localization energies for individual carriers and bound electron-hole pairs.

\section{B. Manipulation and saturation of the PPC}

Another way to transfer carriers trapped in RLPF is by optical excitation using light with an energy larger than $E_{\text {rec }}$ as shown schematically in Fig. 5(b). We demonstrated in Fig. 3 that for photon energies below the effective band gap of the QW no extra carriers are generated and, therefore, no PPC can build up. However, if carriers are already present in the system and trapped in RLPF they can be activated giving rise to a response in the PPC. We want to note that the ability to manipulate the PPC is a clear indication that the underlying mechanism in our II-VI QW-system has a different origin, e.g., in AlGaAs where PPC is dominated by DX-centers. To confirm this assumption a second laser (Laser-2) with an energy $\hbar \omega_{e x}=1.84 \mathrm{eV}$ less than the effective band gap of the QW was used for excitation. Due to its smaller energy no excitons are generated by this laser via interband absorption. Figure 6 shows in the main panel the attenuation of the first SAW harmonic over time for a temperature of $T=45 \mathrm{~K}$, which ensures a linear dependency on the conductivity. At $t=0 \mathrm{~s}$ the above band gap excitation $\hbar \omega_{e x}=3.18 \mathrm{eV}$ of Laser-1 is turned off (gray shaded area) and the SAW attenuation decays slowly as described before. At $t=3.5 \mathrm{~s}$, Laser-2 is turned on and a sharp increase in the SAW attenuation is

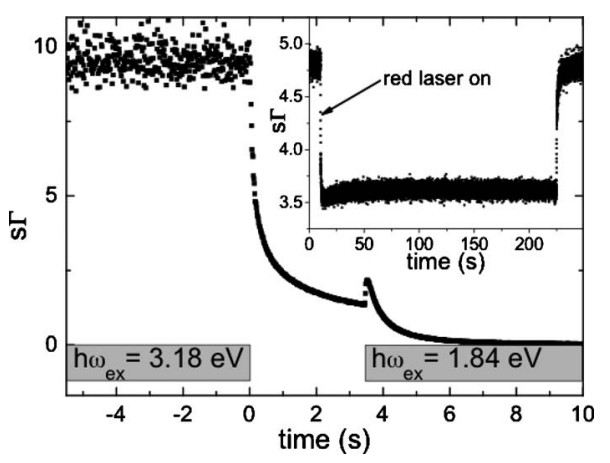

FIG. 6. Manipulation of the attenuation after the excitation with above bandgap energy ( $T=45 \mathrm{~K}$; first SAW harmonic). Irradiation time of the different photon energies is indicated by bars. Inset: Manipulation of the attenuation by optical excitation with $\hbar \omega=1.84 \mathrm{eV}$ during above bandgap excitation ( $T=60 \mathrm{~K}$; first SAW harmonic).

observed. This increase can be understood and explained by an intraband activation of trapped electrons and holes as shown schematically in Fig. 5(b). After activation these carriers now contribute to the sheet conductivity until they recombine. This gives rise to the observed decay of the conductivity even though Laser-2 remains on. This is furthermore consistent with the fact that no additional electrons and holes are photogenerated and only trapped carriers are activated which are lost after recombination. Clearly, the time constant of the decay is faster with Laser- 2 switched on which was also observed using an infrared light emitting diode instead of Laser-2. To further confirm this additional activation effect we performed a steady state experiment shown in the inset of Fig. 6. Here, Laser-1 is on all the time and at $t=10 \mathrm{~s}$ also Laser-2 is used to excite the sample. When Laser-2 is switched on, the SAW attenuation $s \Gamma$ drops by $\sim 25 \%$ since additional activation and subsequent recombination of carriers trapped in RPFL occurs. After Laser-2 is switched off again the original attenuation level is recovered.

To further support our model based on RLPF we performed SAW attenuation experiments under continuous excitation using Laser-1 in which the optical pump power was varied and thus, the generation rate of excitons. Assuming that the rates of radiative decay of excitons and recombination of charges trapped in RLPF are independent on the optical pump power, a steady state occupation of RLPF and number of mobile carriers will build up for a given optical power. The measured attenuation as a function of the excitation intensity is shown as symbols in Fig. 7. With increasing laser intensity the SAW attenuation of the third harmonic and, therefore, the sheet conductivity rises and at about $I$ $\approx 0.1 \times I_{0}$, the sheet conductivity matches the characteristic conductivity. The observed behavior can be fitted in this excitation power range using

$$
s \Gamma\left(I / I_{0}\right)=\frac{K_{\mathrm{eff}}^{2} \cdot s \cdot k}{2} \frac{\frac{\sigma\left(I / I_{0}\right)}{\sigma_{m}}}{1+\left[\frac{\sigma\left(I / I_{0}\right)}{\sigma_{m}}\right]^{2}},
$$

and assuming for $\sigma$ a single exponential increase given by $\sigma\left(I / I_{0}\right) \propto \exp \left(I / I_{0}\right)$. Clearly, the result of this fit (solid line in Fig. 7) agrees well over a wide range of intensities. The 


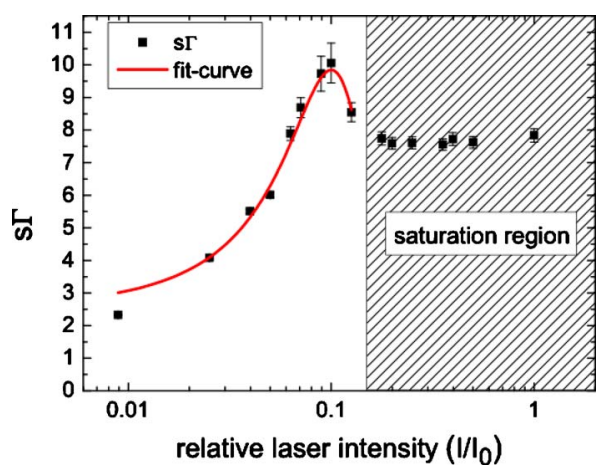

FIG. 7. (Color online) Attenuation of the SAW third harmonic vs laser intensity at $T=60 \mathrm{~K}$. The standard deviation of the attenuation during illumination is indicated by error bars. The solid line represents a fit to the experimental data of Eq. (4).

initial increase in the conductivity can be well understood by assuming a Gaussian variation in the Cd-content from the nominal value of 0.1 and, thus, a Gaussian distribution of $E_{\text {rec }}$. Furthermore, this distribution can be correlated with the distribution of the electron kinetic energy in the RLPF. At $T=60 \mathrm{~K}$ the conduction is in the percolation state and, therefore, at low laser intensities the small number of generated electrons is mainly trapped by deep localization centers with an increased $\mathrm{Cd}$-content, minimizing their kinetic energy. With increasing excitation intensity and electron density, electrons have to populate the shallower localization centers with reduced Cd-content. This leads to a strong increase in total kinetic energy of the trapped electrons and explains the exponential rise of sheet conductivity. The overall good agreement between the experimental data and the fit over a wide range of excitation power proves both the validity of Eq. (1) and the assumption made for $\sigma\left(I / I_{0}\right)$. In addition, the observed saturation of the signal is a clear indication to a finite number of localization site available which are fully populated for laser intensities $>0.2 \times I_{0}$.

At this point we want to note that the applied SAWtechnique is capable to sensitively detect conductivity at very low optical pump powers. In particular we want to note that the minimum excitation power required to detect PL signals from our samples was in the order of $\sim 0.05 \times I_{0}$ $\sim 200 \mathrm{~mW} \mathrm{~cm}^{-2}$ setting the lower bound at which this invasive method can be applied. In contrast, at this particular pump power already a pronounced SAW attenuation of $s \Gamma$ $=6$ is detected. This attenuation level is a factor of 20-30 larger than minimum resolvable attenuation levels of $0.2 \pm 0.1$. We estimate this value from the baseline and noise level in of data in Fig. 3 measured for photon energies less than the effective band gap of the QWs. We extrapolate that at this optical excitation levels $<4 \times 10^{14}$ photons $/ \mathrm{s} \mathrm{cm}^{2}$ are absorbed in the $\mathrm{ZnSe}$ thin epitaxial film. ${ }^{26}$ In addition to this high sensitivity our SAW attenuation method does not rely on high SAW powers. In particular it is capable to detect in the low SAW power regime where no influence on the PL signal is detected or charge conveyance effects occur. Thus, this method does not perturb the carrier system in study and resembles a noninvasive probe of the conductivity. In addition, we have shown that despite of the nonlinear depen- dence on the conductivity given by Eq. (1) the SAWresponse on the conductivity can be analyzed and fitted accurately (Fig. 7).

\section{CONCLUSIONS}

In conclusion we studied the attenuation of a SAW in a $\mathrm{ZnCdSe} / \mathrm{ZnSe}-\mathrm{LiNbO}_{3}$-hybrid. Under optical excitation we observe a variation in the SAW attenuation due to PPC effects in the ternary II-VI alloy. One major advantage of our SAW-based approach is that it does not require electric contacts to the carrier system in study. Moreover, we want to point out that for the presented method an epitaxial lift-off is not necessarily required. Such conductivity measurements can also be also performed by bringing the sample in contact to the $\mathrm{LiNbO}_{3}$ substrate. ${ }^{27}$ This PPC arises from carriers trapped in and activated from random potential fluctuations due to compositional fluctuations in the material. By performing temperature and excitation energy and power dependent experiments impurity or defect related effects can be excluded. It is shown that the carriers trapped in RLPF can be activated optically and thermally. In the temperature dependent experiments a clear transition from a hopping to a percolation regime is observed which also manifests itself in a suppression of bound exciton emission. We find that the critical temperature below which localization occurs is reduced from $T=120 \mathrm{~K}$ for bulk crystals to $T<40 \mathrm{~K}$ in high quality QWs grown by MBE. The corresponding shallow potential modulation of $E_{\text {rec }}=\$ 3 \mathrm{meV}$ underlines the high structural quality achievable in MBE-grown II-VI heterostructures. Furthermore, direct comparison between the applied techniques demonstrates that compared to PL spectroscopy the SAW-based method yields a very versatile, noninvasive tool for sensitive conductivity measurements which can be applied to other material systems than the one studied in this work.

This work was supported in parts by the German federal government as part of the Cluster of Excellence Nanosystems Initiative Munich (NIM). The work in the UK was funded by EU (SANDiE) and by EPSRC.

${ }^{1}$ A. Wixforth, J. Scriba, M. Wassermeier, J. P. Kotthaus, G. Weimann, and W. Schlapp, Phys. Rev. B 40, 7874 (1989).

${ }^{2}$ M. Rotter, A. V. Kalameitsev, A. O. Govorov, W. Ruile, and A. Wixforth, Phys. Rev. Lett. 82, 2171 (1999).

${ }^{3}$ C. Rocke, A. O. Govorov, A. Wixforth, G. Böhm, and G. Weimann, Phys. Rev. B 57, R6850 (1998).

${ }^{4}$ O. D. D. Couto, Jr., F. Iikawa, J. Rudolph, R. Hey, and P. V. Santos, Phys. Rev. Lett. 98, 036603 (2007).

${ }^{5}$ C. Bödefeld, J. Ebbecke, J. Toivonen, M. Sopanen, H. Lipsanen, and A. Wixforth, Phys. Rev. B 74, 035407 (2006).

${ }^{6}$ J. R. Gell, M. B. Ward, R. J. Young, R. M. Stevenson, P. Atkinson, D. Anderson, G. A. C. Jones, D. A. Ritchie, and A. J. Shields, Appl. Phys. Lett. 93, 081115 (2008).

${ }^{7}$ O. D. D. Couto, Jr., S. Lazic, F. Iikawa, J. A. H. Stotz, U. Jahn, R. Hey, and P. V. Santos, Nat. Photonics 3, 645 (2009).

${ }^{8}$ D. A. Fuhrmann, A. Wixforth, A. Curran, J. K. Morrod, K. A. Prior, R. J. Warburton, and J. Ebbecke, Appl. Phys. Lett. 94, 193505 (2009).

${ }^{9}$ D. V. Lang, R. A. Logan, and M. Jaros, Phys. Rev. B 19, 1015 (1979).

${ }^{10}$ F. Firszt, H. Meczynska, S. Legowski, J. Zakrzewski, K. Strzalkowski, and M. Wrobel, Phys. Status Solidi C 1, 916 (2004).

${ }^{11}$ S. Scholl, J. Gerschütz, H. Schäfer, F. Fischer, A. Waag, and G. Landwehr, Solid State Commun. 91, 491 (1994).

${ }^{12}$ M. K. Sheinkman and A. Y. Shik, Sov. Phys. Semicond. 10, 128 (1976). 
${ }^{13}$ H. J. Queisser and D. E. Theodorou, Phys. Rev. B 33, 4027 (1986).

${ }^{14}$ A. S. Dissanayake, S. X. Huang, H. X. Jiang, and J. Y. Lin, Phys. Rev. B 44, 13343 (1991).

${ }^{15}$ A. Dissanayake, M. Elahi, H. X. Jiang, and J. Y. Lin, Phys. Rev. B 45, 13996 (1992)

${ }^{16}$ H. X. Jiang and J. Y. Lin, Phys. Rev. B 40, 10025 (1989).

${ }^{17}$ H. X. Jiang and J. Y. Lin, Phys. Rev. Lett. 64, 2547 (1990).

${ }^{18}$ H. X. Jiang, G. Brown, and J. Y. Lin, J. Appl. Phys. 69, 6701 (1991).

${ }^{19}$ H. X. Jiang, A. Dissanayake, and J. Y. Lin, Phys. Rev. B 45, 4520 (1992).

${ }^{20}$ J. Y. Lin and H. X. Jiang, Phys. Rev. B 41, 5178 (1990).

${ }^{21}$ C. W. Chang, H. C. Yang, C. H. Chen, H. J. Chang, and Y. F. Chen, J.
Appl. Phys. 89, 3725 (2001).

${ }^{22}$ A. Balocchi, A. Curran, T. C. M. Graham, C. Bradford, K. A. Prior, and R. J. Warburton, Appl. Phys. Lett. 86, 011915 (2005).

${ }^{23}$ S. H. Simon, Phys. Rev. B 54, 13878 (1996).

${ }^{24}$ F. Liaci, P. Bigenwald, O. Briot, B. Gil, N. Briot, and T. A. Cloitre, Phys. Rev. B 51, 4699 (1995).

${ }^{25}$ G. E. Hite, D. T. F. Marple, M. Aven, and B. Segall, Phys. Rev. 156, 850 (1967).

${ }^{26}$ S. Adachi and T. Taguchi, Phys. Rev. B 43, 9569 (1991).

${ }^{27}$ A. Wixforth, J. Scriba, M. Wassermeier, J. P. Kotthaus, G. Weimann, and W. Schlapp, J. Appl. Phys. 64, 2213 (1988). 